\title{
Determination of complete chromosomal haplotypes by bulk DNA sequencing
}

\author{
Richard W. Tourdot ${ }^{\mathrm{a}, \mathrm{b}, \mathrm{c}}$ and Cheng-Zhong Zhang $\mathrm{g}^{\mathrm{a}, \mathrm{b}, \mathrm{c}}$ \\ ${ }^{a}$ Department of Data Sciences, Dana-Farber Cancer Institute, 3 Blackfan Circle, Boston MA 02215 \\ ${ }^{\mathrm{b}}$ Department of Biomedical Informatics, 10 Shattuck Street, Harvard Medical School, Boston MA 02115 \\ ${ }^{\mathrm{c}}$ Cancer Program, Broad Institute of MIT and Harvard, 415 Main Street, Cambridge MA 02142
}

June 20, 2020

\begin{abstract}
Haplotype phase represents the collective genetic variation between homologous chromosomes and is an essential feature of nonhaploid genomes. Determining the haplotype phase requires knowledge of both the genotypes at variant sites and their linkage across each chromosome. Haplotype linkage can be either inferred statistically from a genotyped population, or determined by long-range sequencing of an individual genome. However, extending haplotype inference to the whole-chromosome scale remains challenging and usually requires special experimental techniques. Here we describe a general computational strategy to determine complete chromosomal haplotypes using a combination of bulk long-range sequencing and $\mathrm{Hi}-\mathrm{C}$ sequencing. We demonstrate that this strategy can resolve the haplotypes of parental chromosomes in diploid human genomes at high precision (99\%) and completeness (98\%), and is further able to assemble the syntenic organization of aneuploid genomes ("digital karyotype").
\end{abstract}

Haplotype phase ("haploid genotype") is the combination of genotypes at sites of genetic variation along a chromosome (The International HapMap Consortium 2005). Knowledge of chromosomal haplotypes is required for interrogating both the differences between homologous chromosomes, such as variations in the DNA sequence and in epigenetic features, and their impacts on gene expression (Shendure and Aiden 2012, Adey et al., 2013a, Hansen and van Oudenaarden, 2013, Levesque and Raj, 2013, Deng et al. 2014, Snyder et al. 2015). Haplotype information is also needed for performing diploid genome assembly (Pendleton et al. 2015; Seo et al. 2016) and can significantly improve the precision and resolution of mutation detection in polyclonal populations (Nik-Zainal et al., 2012, Loh et al., 2018) or single cells (Zhang and Pellman, 2015).

There are two strategies of haplotype inference (Browning and Browning, 2011, Snyder et al. 2015). The first strategy ("statistical phasing") (Scheet and Stephens, 2006, Browning and Browning 2007, Loh et al., 2016a) infers haplotype phase based on the recombination probabilities between variant genotypes estimated from linkage disequilibrium in a population (Reich et al. 2001; Daly et al. 2001). Although statistical phasing can infer haplotype linkage between adjacent variant sites at reasonably high accuracy (99\%), the accumulation of random switching errors $(0.1 \%)$ precludes long-range (10Mb) haplotype inference unless the genotypes of closely related individuals are used (Kong et al. 2008, Loh et al. 2016b). Statistical phasing is also restricted to common polymorphisms and not applicable to de novo mutations.

The second strategy directly extracts haplotype linkage from the DNA sequences of single chromosomes or sub-haploid chromosomal fragments ("molecular linkage") (Snyder et al. 2015). Direct sequencing of single chromosomes can produce wholechromosome haplotypes (Ma et al., 2010, Fan et al. 2010, Yang et al. 2010, Zhang et al. 2015, Porubsky et al. 2017) but is only applicable to dividing cells and requires laborious experimental procedures of chromosome isolation or tagging. Long-read sequencing or long-range sequencing can either reveal haplotype linkage directly from long contiguous reads $(\sim 10 \mathrm{~kb})$, or indirectly from short DNA fragments derived from long DNA molecules $(\sim 100 \mathrm{~kb})$ that are tagged with unique molecular barcodes (Kitzman et al. 2010, Suk et al. 2011, Kaper et al. 2013, Adey et al. 2013b 2014, Peters et al. 2014, Zheng et al., 2016, Chu et al. 2017, Marks et al. 2019). The typical size of DNA molecules in long-read or long-range sequencing (10-100kb) is sufficient for linking variants in regions of normal variant density ( $\sim 1$ per kb), but inadequate in regions of low variant density $(1 \mathrm{per} 10 \mathrm{~kb})$, and unable to bridge large gaps $(100 \mathrm{~kb})$ with no identifiable variants, including all centromeres.

Molecular linkage across individual chromosomes ( cis linkage) is also contained in Hi-C contacts generated by proximitybased chromatin ligation (Rao et al. 2014). As chromosomes are spatially isolated in separate territories in the cell nucleus, $\mathrm{Hi}-\mathrm{C}$ fragments are predominantly formed within the same chromosome and Hi-C sequencing can preserve intra-chromosomal linkage extending to the whole chromosome (Selvaraj et al. 2013) without single-chromosome isolation. The main shortcoming of haplotype linkage from Hi-C data is its sparsity: Only a small fraction of genetic variants are covered and linked by long-range Hi-C contacts; the sparse linkage cannot produce contiguous haplotypes (Edge et al. 2017) except for genomes with an exceptionally high variant density ( $\sim 1$ per 150 bp) (Selvaraj et al. 2013). The sparsity of long-range Hi-C linkage also limits its utility for phasing de novo mutations. 
bioRxiv preprint doi: https://doi.org/10.1101/629337; this version posted June 21,2020 . The copyright holder for this preprint (which was not certified by peer review) is the author/funder, who has granted bioRxiv a license to display the preprint in perpetuity. It is made available under aCC-BY-NC-ND 4.0 International license.

Although Hi-C contacts are too sparse to link single genetic variants, they generate sufficient linkage to concatenate haplotype blocks each consisting of hundreds of variants. This idea has led us to design a two-tier approach to determine complete wholechromosome haplotypes combining long-range sequencing and $\mathrm{Hi}-\mathrm{C}$ sequencing (Figure 1). This approach first determines local haplotype blocks consisting of $10^{2}-10^{3}$ variants using long-range sequencing and then merge these blocks into a single haplotype using Hi-C contacts between blocks. We formulate both local haplotype inference and haplotype block concatenation as a minimization problem that can be efficiently solved by steepest descent methods. Applying our approach to two diploid human samples with reference haplotype data, we demonstrate that the computational inference produces the haplotypes of parental chromosomes with high accuracy (99\%) and completeness (98\%) and having no long-range switching error. We then describe strategies to analyze structural alterations of chromosomes using the parental haplotype, including the assembly of derivative chromosomes in aneuploid genomes using chromosome-specific Hi-C contacts.

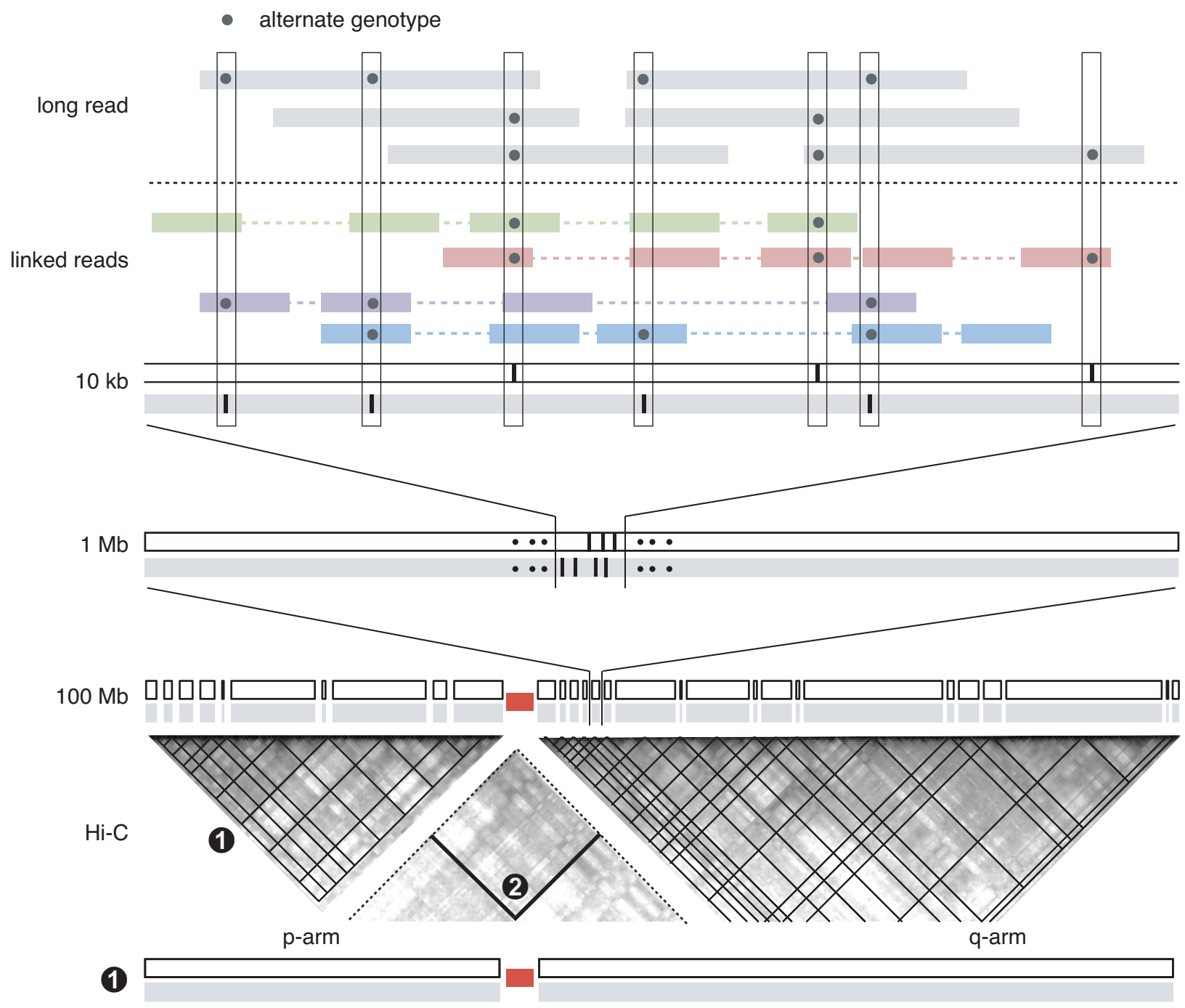

(2)

Figure 1: A hierarchical strategy for determining whole chromosome haplotypes. Megabase-scale haplotype blocks are determined using linkage information from linked-reads or long-read sequencing and then concatenated using Hi-C contacts, first within each chromosome arm and then between the $\mathrm{p}$ - and q-arms. 


\section{Results}

\section{Data generation and processing}

For the computational inference and benchmarking of parental haplotypes in diploid genomes, we used published and newly generated datasets of the NA12878 cell line and the retinal pigment epithelium (RPE-1) cell line. Data sources are listed in Table 1 . For the analysis of altered chromosomes in aneuploid genomes, we used bulk whole-genome sequencing data of aneuploid RPE-1 cells from Ref. (Maciejowski et al., 2015) and published cytogenetic (Gribble et al., 2000, Naumann et al. 2001) and sequencing (linked-reads and Hi-C) data of K-562 cells (Table S1). A detailed description of the experimental and data-processing protocols is provided in SI Materials and Methods.

Table 1: Sources of data for parental haplotype inference and benchmarking

\begin{tabular}{|c|c|c|c|c|c|c|}
\hline Sample & Data type & Data source & Read count & $\begin{array}{l}\text { Mean } \\
\text { depth }\end{array}$ & $\begin{array}{r}\text { Contacts } \\
(1 \mathrm{Mb})\end{array}$ & Application \\
\hline RPE-1 & bulk WGS & Zhang et al. 2015 & $228,708,76$ & $13 \times$ & & variant calling \\
\hline RPE-1 & linked reads & new & $941,518,42 c^{b}$ & $60 \times c$ & & $\begin{array}{l}\text { variant calling } \\
\& \text { local phasing }\end{array}$ \\
\hline RPE-1 & $\mathrm{Hi}-\mathrm{C}$ & Darrow et al. (2016) & $281,285,484^{d}$ & & $48,124,211$ & long-range phasing \\
\hline RPE-1 & $\begin{array}{r}\text { single cell with } \\
\text { monosomies }\end{array}$ & new & & & & $\begin{array}{l}\text { hi-conf variants \& } \\
\text { reference haplotypes }\end{array}$ \\
\hline NA12878 & linked reads v.1 & 10X Genomic $e$ & $422,179,399 f$ & $35 \sqrt{ } \times$ & & local phasing \\
\hline NA12878 & linked reads v. 2 & 10X Genomic & $423,854,243^{n}$ & $35 \times \sqrt{c}$ & & local phasing \\
\hline NA12878 & $\mathrm{Hi}-\mathrm{C}$ & Rao et al. (2014) & $486,848,169^{t}$ & & $91,428,507$ & long-range phasing \\
\hline NA12878 & phased VCF & GIAB $^{j}$ & & & & $\begin{array}{l}\text { hi-conf variants \& } \\
\text { reference haplotypes }\end{array}$ \\
\hline
\end{tabular}

${ }^{a}$ SRR1778442: median insert $243 ; 208,151,992$ aligned in pair; $101 \times 2$; duplication rate 0.024 .

${ }^{b}$ Mean molecular length $24.8 \mathrm{~kb}$; median insert $551 ; 913,660,083$ aligned in pair; $150 \times 2$; duplication rate 0.255 .

${ }^{c}$ excluding the GEMcode sequence

${ }^{d}$ SRS1045722: median insert 364; 279,027,892 aligned in pair; $150 \times 2$; duplication rate 0.067 .

' https://support.10xgenomics.com/genome-exome/datasets/2.1.0/NA12878_WGS_210

${ }^{j}$ Mean molecular length $68.7 \mathrm{~kb}$; median insert 349; 407,015,530 aligned in pair; $150 \times 2$; duplication rate 0.062 .

$\varepsilon$ https://support.10xgenomics.com/genome-exome/datasets/2.2.1/NA12878_WGS_v2

${ }^{h}$ Mean molecular length $85.6 \mathrm{~kb}$; median insert 370; 418,283,435 aligned in pair; $150 \times 2$; duplication rate 0.079 .

${ }^{i}$ SRR1658572: median insert 377; 484,211,662 aligned in pair; $101 \times 2$; duplication rate 0.028 .

https://ftp-trace.ncbi.nlm.nih.gov/ReferenceSamples/giab/release/NA12878_HG001/latest/GRCh38/

\section{Hi-C sequencing can reveal haplotype linkage across entire chromosomes}

The basic unit of haplotype linkage evidence is a molecular link, which we use to denote a single DNA molecule providing genotype information at multiple variant sites. In long-read sequencing, a link consists of a single contiguous alignment; in Hi-C data, a link consists of multiple alignments of a Hi-C fragment; in linked-reads sequencing, a link consists of a group of sequencing fragments sharing the same molecular barcode. To demonstrate the utility of Hi-C sequencing for long-range haplotype inference, we calculate three metrics of haplotype linkage between heterozygous variant sites at different genomic distance: (1) percentage of linked variants; (2) average number of links between linked variants; and (3) percentage of links consistent with cis linkage according to the reference parental haplotype. These results are shown in Fig. 2 together with the same metrics of haplotype linkage from linked-reads data for comparison.

The density of Hi-C links shows a power-law decay against genomic distance (Fig. 2A,B) that is similar to the probability of intra-chromosomal contacts (Lieberman-Aiden et al., 2009, Sanborn et al. 2015). The power-law dependence suggests that most Hi-C links result from intra-chromosomal contacts, as the presence of inter-chromosomal contacts (which should form largely at random) will cause deviations from the power-law decay. This is validated by the observation that more than $90 \%$ of all $\mathrm{Hi}-\mathrm{C}$ links are consistent with cis contacts between loci in the same chromosome (Fig. $2 \mathrm{E}, \mathrm{F}$ ). By contrast, haplotype linkage from the linked-reads data has a limited range determined by the maximum size of input DNA molecules $(\sim 100 \mathrm{~kb}$ in the RPE-1 library and $\sim 300 \mathrm{~kb}$ in the NA12878 libraries). The distance-independent linkage signal beyond the molecular size most likely arises from unrelated DNA fragments being tagged by the same molecular barcode by chance: As the unrelated DNA fragments can originate from either parental chromosomes, the average percentage of apparent cis or trans linkage is 50\% (Fig. 2E,F), despite all links being intermolecular.

Although Hi-C links across the entire chromosome are dominated by intra-molecular Hi-C contacts, the sparsity of long-range $\mathrm{Hi}-\mathrm{C}$ contacts limits its utility for haplotype inference. In both Hi-C data (RPE-1 and NA12878), the probability that two variant 
A
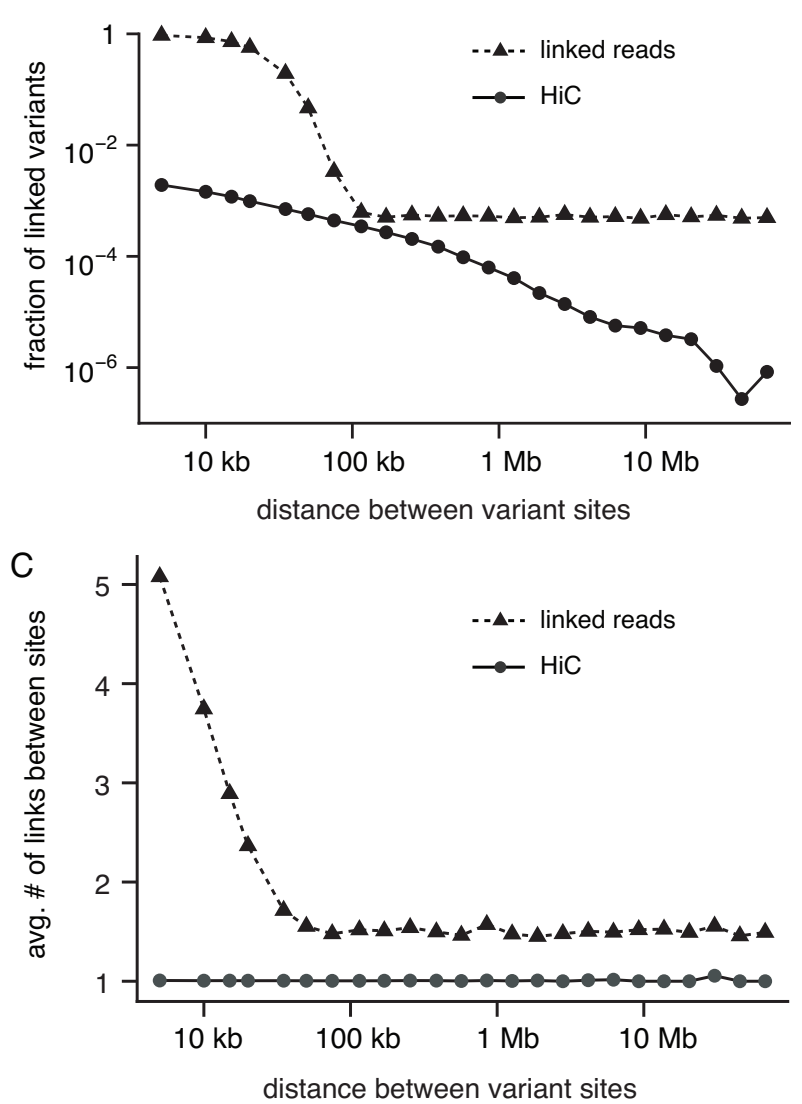

$\mathrm{E}$

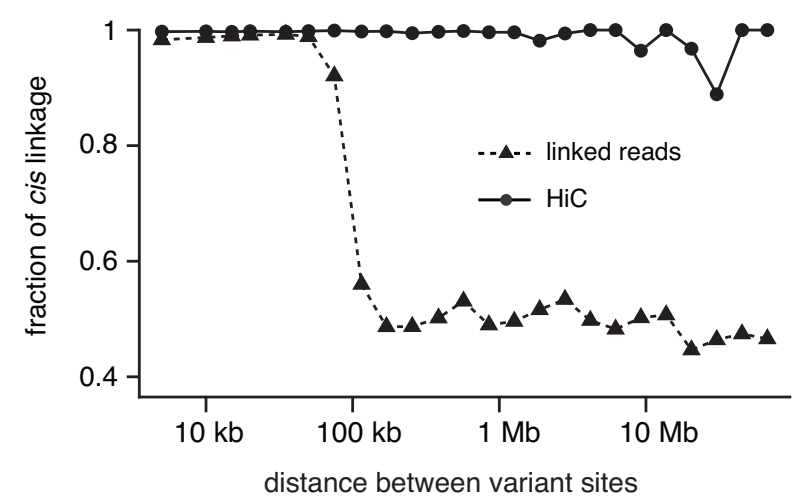

B

NA12878
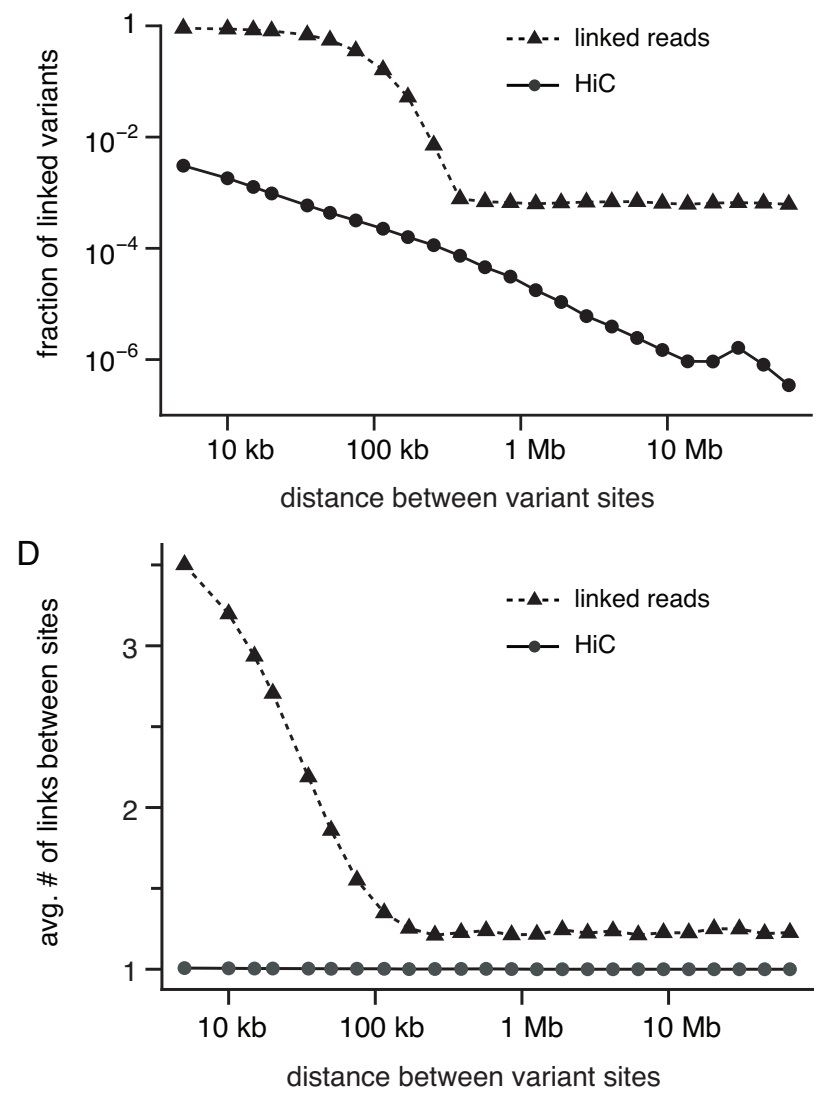

$\mathrm{F}$

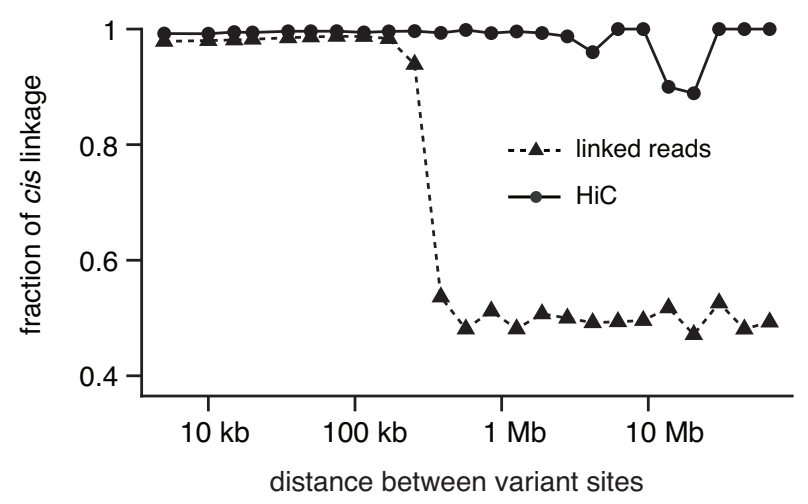

Figure 2: Statistical metrics of haplotype linkage between variants at different genomic distance extracted from linked-reads and Hi-C sequencing data of RPE-1 (A,C,E) and NA12878 (B,D,F) cells. A and B. Fraction of linked variants; C and D. Average number of links between linked variants; E and F. Fraction of links consistent with cis linkage. The residual cis linkage in linkedreads data $(\sim 50 \%)$ reflects unrelated DNA fragments from either parental homologs being tagged by identical molecular barcodes by chance.

sites separated by $100 \mathrm{~kb}$ are linked by Hi-C reads is less than $10^{-3}$ (Fig. 2A,B) and almost all linkage consists of only one link/read (Fig. 2 2 C,D). Such weak linkage evidence is inadequate for accurate haplotype inference at all variant sites.

To take advantage of long-range Hi-C linkage without significantly increasing the number of $\mathrm{Hi}-\mathrm{C}$ reads, we can aggregate $\mathrm{Hi}-\mathrm{C}$ 
links between local haplotype blocks consisting of $10^{2}$ or more variants to generate a specific linkage signal between these blocks. To demonstrate this quantitatively, we calculate the average number of $\mathrm{Hi}-\mathrm{C}$ links between $0.5,1$, and $2 \mathrm{Mb}$ segments at different genomic distance (Fig. 34). This calculation shows that the Hi-C linkage signal between megabase-scale segments can extend well above $10 \mathrm{Mb}$, which is sufficient to merge all haplotype blocks on each chromosome. As haplotype inference by either statistical phasing or long-range sequencing can conveniently generate megabase-scale haplotype blocks, we can use Hi-C sequencing only for linking these blocks across low-variant density regions, including gaps in the reference genome. We next describe a general computational strategy of haplotype inference that is applicable to both local haplotype inference and haplotype block concatenation (Fig. 3 B $)$.

\section{Haplotype inference from linkage evidence}

We first introduce a binary numerical representation of genotypes at heterozygous variants as 1 for the reference base and -1 for the alternate base. A haplotype block consisting of $N$ variant sites is represented as a vector

$$
\mathbf{S}\left(s_{1}, s_{2}, \cdots, s_{N}\right), \quad s_{i} \pm 1 .
$$

Similarly, a molecular link with genotype information at multiple variant sites is represented as

$$
\boldsymbol{\sigma}\left(\sigma_{1}, \sigma_{2}, \cdots\right), \quad \sigma_{i} \pm 1
$$

Using the binary genotype representation, we can simply four types of linkage between genotypes (for both $\sigma_{i}$ and $s_{i}$ )

$$
\begin{array}{ll}
\text { reference-reference linkage: } & s_{i} 1, s_{j} 1 ; \\
\text { alternate-alternate linkage: } & s_{i}-1, s_{j}-1 ; \\
\text { reference-alternate linkage: } & s_{i} 1, s_{j}-1 ; \\
\text { alternate-reference linkage: } & s_{i}-1, s_{j} 1 .
\end{array}
$$

into two types of haplotype linkage

reference-reference/alternate-alternate linkage: $s_{i} \cdot s_{j} 1$, reference-alternate/alternate-reference linkage: $s_{i} \cdot s_{j}-1$.

Moreover, a molecular link $\left(\sigma_{i}, \sigma_{j}\right)$ between sites $i$ and $j$ is consistent with haplotype linkage $\left(s_{i}, s_{j}\right)$ if and only if

$$
\sigma_{i} \sigma_{j} s_{i} s_{j} 1
$$

A

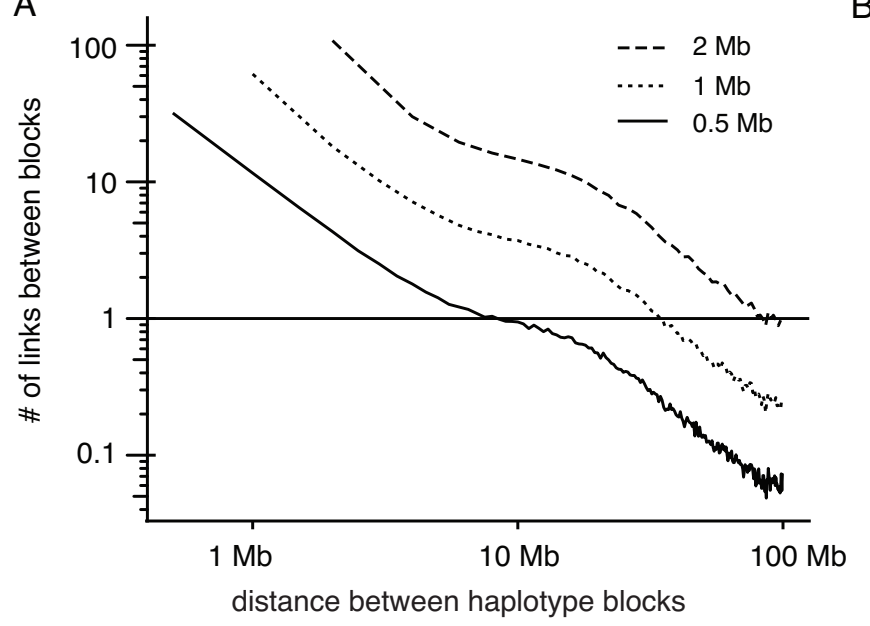

B

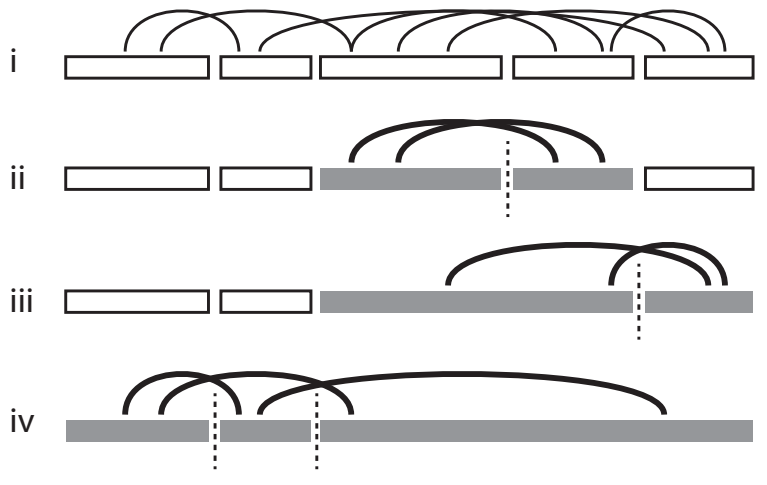

Figure 3: Linking haplotype blocks with Hi-C reads. A. Average number of Hi-C links between two segments of $0.5,1$, and $2 \mathrm{Mb}$ at different genomic distance calculated using the RPE-1 Hi-C data. B. Concatenation of small haplotype blocks using Hi-C reads. (i) Local haplotype blocks (open rectangles) and Hi-C links (curves); (ii) and (iii) merging of adjacent haplotype blocks using Hi-C links; (iv) joint inference of the haplotype phase of all blocks using all Hi-C links. 
If the error probability of a molecular link is given by $\epsilon_{i j}$, then

$$
p\left(\sigma_{i} \sigma_{j} s_{i} s_{j} 1\right) 1-\epsilon_{i j} ; \quad p\left(\sigma_{i} \sigma_{j} s_{i} s_{j}-1\right) \epsilon_{i j} .
$$

Assuming $p\left(s_{i} s_{j} 1\right) p\left(s_{i} s_{j}-1\right) 1 / 2$, we can re-write the above equation as

$$
\frac{p\left(\sigma_{i} \sigma_{j} \mid s_{i} s_{j} 1\right)}{p\left(\sigma_{i} \sigma_{j} \mid s_{i} s_{j}-1\right)}\left(\frac{1-\epsilon_{i j}}{\epsilon_{i j}}\right)^{\sigma_{i} \sigma_{j} s_{i} s_{j}} .
$$

Extending this to a collection of links $\left\{\sigma_{i}^{(k)} \sigma_{j}^{(k)}, 1 \leq k \leq n\right\}$, we have

$$
\frac{p\left(\sigma_{i}^{(k)} \sigma_{j}^{(k)} \mid s_{i} s_{j} 1\right)}{p\left(\sigma_{i}^{(k)} \sigma_{j}^{(k)} \mid s_{i} s_{j}-1\right)} \prod_{k}\left(\frac{1-\epsilon_{i j}^{(k)}}{\epsilon_{i j}^{(k)}}\right){ }^{(k)} \sigma_{j}^{(k)} s_{i} s_{j}
$$

which leads to the following log-likelihood function

$$
\begin{gathered}
L\left(\left\{\sigma_{i}^{(k)} \sigma_{j}^{(k)}\right\} \mid s_{i} s_{j}\right) \ln p\left(\sigma_{i}^{(k)} \sigma_{j}^{(k)} \mid s_{i} s_{j} 1\right)-\ln p\left(\sigma_{i}^{(k)} \sigma_{j}^{(k)} \mid s_{i} s_{j}-1\right) \\
\sum_{k} \sigma_{i}^{(k)} \sigma_{j}^{(k)} s_{i} s_{j} \ln \left(1-\epsilon_{i j}^{(k)} / \epsilon_{i j}^{(k)}\right)
\end{gathered}
$$

If we assume a constant error rate for all links, $\epsilon_{i j}^{(k)} \epsilon$, Eq. 2 is simplified to

$$
\begin{aligned}
L\left(\left\{\sigma_{i}^{(k)} \sigma_{j}^{(k)}\right\} \mid s_{i} s_{j}\right) & \ln \left(\frac{1-\epsilon}{\epsilon}\right) \sum_{k} \sigma_{i}^{(k)} \sigma_{j}^{(k)} s_{i} s_{j} \\
& \propto \underbrace{\#\left(\sigma_{i} \sigma_{j} s_{i} s_{j} 1\right)}_{\text {consistent links }}-\underbrace{\#\left(\sigma_{i} \sigma_{j} s_{i} s_{j}-1\right)}_{\text {inconsistent links }} .
\end{aligned}
$$

The haplotype linkage inferred from all molecular links is given by

$$
s_{i} s_{j} \begin{cases}1 & \sum_{k} \sigma_{i}^{(k)} \sigma_{j}^{(k)} 0 \\ -1 & \sum_{k} \sigma_{i}^{(k)} \sigma_{j}^{(k)}\end{cases}
$$

We can generalize Eq. (2) to $N$ variants as

$$
L\left(\left\{\boldsymbol{\sigma}^{(k)}\right\} \mid \mathbf{S}\right) \frac{1}{2} \sum_{1 \leq i, j \leq N} s_{i} s_{j} \sum_{k} \sigma_{i}^{(k)} \sigma_{j}^{(k)} \ln \left(1-\epsilon_{i j}^{(k)} / \epsilon_{i j}^{(k)}\right),
$$

and solve for the optimal haplotype solution $\$$ by maximizing Eq. 5 (assuming a uniform prior probability $p(\mathbf{S})$ ). We further assume the frequency of incorrect linkage is constant between different molecules but can vary between variant sites, $\epsilon_{i j}^{(k)} \epsilon_{i j}$. We can then rewrite Eq. [5] as

$$
\begin{aligned}
L\left(\left\{\sigma^{(k)}\right\} \mid \mathbf{S}\right) & \frac{1}{2} \sum_{1 \leq i, j \leq N} s_{i} s_{j} \ln \left(1-\epsilon_{i j} / \epsilon_{i j}\right) \sum_{k} \sigma_{i}^{(k)} \sigma_{j}^{(k)} \\
& \frac{1}{2} \sum_{1 \leq i, j \leq N} s_{i} s_{j} \ln \left(1-\epsilon_{i j} / \epsilon_{i j}\right)\left(n_{i j}-n_{i j}^{-}\right),
\end{aligned}
$$

with the introduction of

$$
\begin{gathered}
n_{i j} \#\left(\sigma_{i}^{(k)} \sigma_{j}^{(k)} 1\right) n_{i j}^{\mathrm{RR}} n_{i j}^{\mathrm{AA}}, \\
n_{i j}^{-} \#\left(\sigma_{i}^{(k)} \sigma_{j}^{(k)}-1\right) n_{i j}^{\mathrm{RA}} n_{i j}^{\mathrm{AR}}
\end{gathered}
$$

corresponding to the number of links supporting each type of haplotype linkage between site $i$ and $j$. We estimate $\epsilon_{i j}$ using

$$
\epsilon_{i j} \max \left[\epsilon_{0}, \frac{\min \left(n_{i j}, n_{i j}^{-}\right)}{n_{i j} n_{i j}^{-}}\right],
$$


where $\min \left(n_{i j}, n_{i j}^{-}\right) /\left(n_{i j} n_{i j}^{-}\right)$is the observed fraction of minor discordant haplotype linkage, and

$$
\epsilon_{0}\left\langle\frac{\min \left(n_{i j}, n_{i j}^{-}\right)}{n_{i j} n_{i j}^{-}}\right\rangle
$$

is the average fraction of observed discordant linkage.

The formalism of haplotype inference defined in Eqs. (5) and (6) has several advantages. First, the binary representation of haplotype phase and molecular linkage preserves the symmetry between parental haplotypes $(\mathbf{S}$ and $-\mathbf{S})$ or molecular links derived from parental chromosomes $(\boldsymbol{\sigma}$ and $-\boldsymbol{\sigma})$. This is convenient for performing haplotype inference in aneuploid genomes where one homolog may contribute dominant linkage evidence (e.g., in hemizygous or trisomic regions).

Second, the formalism is directly applicable to haplotype block phasing. The phase of haplotype blocks $\mathbf{B}_{1}, \mathbf{B}_{2}, \cdots, \mathbf{B}_{m}$ can be represented as a vector

$$
\mathbf{B}\left(b_{1}, b_{2}, \cdots, b_{m}\right), \quad b_{s} \pm 1,
$$

and determined from inter-block molecular linkage (similar to Eq. (7))

$$
\begin{aligned}
& n_{s t} n\left(\mathbf{B}_{s} \leftrightarrow \mathbf{B}_{t}\right) n\left(\overline{\mathbf{B}_{s}} \leftrightarrow \overline{\mathbf{B}_{t}}\right) ; \\
& n_{s t}^{-} n\left(\overline{\mathbf{B}_{s}} \leftrightarrow \mathbf{B}_{t}\right) n\left(\mathbf{B}_{s} \leftrightarrow \overline{\mathbf{B}_{t}}\right) .
\end{aligned}
$$

$\left(\overline{\mathbf{B}_{k}}-\mathbf{B}_{k}\right.$ is the complementary phase block of $\left.\mathbf{B}_{k}.\right)$

Finally, maximizing Eq. 6) is equivalent to minimizing the energy of a 1D Ising model (or spin glass model)

$$
E(\mathbf{S})-\frac{1}{2} \sum_{1 \leq i, j \leq N} M_{i j} s_{i} s_{j}
$$

with finite range interactions $M_{i j}\left(n_{i j}-n_{i j}^{-}\right) \ln \left(1-\epsilon_{i j} / \epsilon_{i j}\right)$, for which there are many existing approaches. Here we solve this problem by introducing two types of perturbations:

$$
\begin{gathered}
\text { spin flip: }\left(\cdots s_{i-1}, s_{i}, s_{i 1}, \cdots\right) \rightarrow\left(\cdots s_{i-1},-s_{i}, s_{i 1}, \cdots\right) \\
\text { block switch: }\left(s_{1}, \cdots s_{i}, s_{i 1}, \cdots s_{N}\right) \rightarrow\left(s_{1}, \cdots s_{i},-s_{i 1}, \cdots-s_{N}\right) .
\end{gathered}
$$

The changes to $E(\mathbf{S})$ due to these perturbations are given by

$$
\Delta E_{i} s_{i} \sum_{j} M_{i j} s_{j}, \quad \text { (spin flip) }
$$

and

$$
\Delta E_{k \mid k 1} \sum_{i \leq k} \sum_{j k} M_{i j} s_{i} s_{j} . \quad \text { (block switch) }
$$

It can be shown that through iterations of spin flipping and block switching, one can always find (one of) the optimal haplotype solutions $\mathbf{S}$ that minimizes Eq. 10 if the majority of molecular linkage is consistent with cis haplotype linkage (SI Discussion).

For two haplotype configurations $\mathbf{S}$ and $\mathbf{S}^{\prime}$, the energy difference is related to the likelihood ratio

$$
\Delta E E(\mathbf{S})-E\left(\mathbf{S}^{\prime}\right) L\left(\sigma^{(k)} \mid \mathbf{S}\right)-L\left(\sigma^{(k)} \mid \mathbf{S}^{\prime}\right) \ln \frac{p\left(\sigma^{(k)} \mid \mathbf{S}\right)}{p\left(\sigma^{(k)} \mid \mathbf{S}^{\prime}\right)} .
$$

Thus, $\Delta E_{i}$ or $\Delta E_{i \mid i 1}$ are related to the probability of phasing errors:

$$
\delta \frac{1}{1 e^{\Delta E}},
$$

A low energy penalty score $(\Delta E \approx 0)$ implies low phasing confidence $(\delta \approx 0.5)$, and vice versa $(\Delta E \gg 0 \Rightarrow \delta \approx 0)$. 
The spin-flipping energy penalty (Eq. [11) can be rewritten as

$$
\begin{gathered}
\Delta E_{i} s_{i} \sum_{j \neq i} M_{i j} s_{j} s_{i} \sum_{j \neq i}\left(n_{i j}-n_{i j}^{-}\right) s_{j} \underbrace{\ln \left(1-\epsilon_{i j} / \epsilon_{i j}\right)}_{\chi_{i j}} \\
s_{i}\left[\sum_{j \neq i, s_{j} 1} \chi_{i j}\left(n_{i j}-n_{i j}^{-}\right)-\sum_{j \neq i, s_{j}-1} \chi_{i j}\left(n_{i j}-n_{i j}^{-}\right)\right] \\
s_{i}(\underbrace{\left.\sum_{\eta_{R} \leftrightarrow \mathbf{S}} \chi_{i j} n_{i j} \sum_{j \neq i, s_{j}-1} \chi_{i j} n_{i j}^{-}\right)}_{j \neq i, s_{j} 1} \\
-s_{i}(\underbrace{\sum_{i}}_{\left.\sum_{j \neq i, s_{j} 1} \chi_{i j} n_{i j}^{-} \sum_{j \neq i, s_{j}-1} \chi_{i j} n_{i j}\right)}
\end{gathered}
$$

The two terms $\eta_{R \leftrightarrow \mathbf{S}}$ and $\eta_{A \leftrightarrow \mathbf{S}}$ in Eq. (14) measure the total linkage between the genotypes at site $i$ ( $R$ for reference and $A$ for alternate) and the haplotypes of parental chromosomes $(\mathbf{S}$ and $-\mathbf{S})$. For true heterozygous variants, reference and alternate genotypes are phased to complementary haplotypes, i.e., $R \leftrightarrow \mathbf{S}$ (and hence $A \leftrightarrow-\mathbf{S})$, or $A \leftrightarrow \mathbf{S}(R \leftrightarrow-\mathbf{S})$. This implies that either $\eta_{R \leftrightarrow \mathbf{S}} \gg \eta_{A \leftrightarrow \mathbf{S}} \approx 0$ or $\eta_{A \leftrightarrow \mathbf{S}} \gg \eta_{R \leftrightarrow \mathbf{S}} \approx 0$. As the genotypes of false variants are generally not phased to complementary haplotypes, false variants tend to have low phasing confidence $(\Delta E \approx 0)$ and can be excluded from the haplotype solution based on this feature. Moreover, linkage evidence from false variants is offset by the $\chi_{i j}$ factor due to the presence of significant discordant linkage evidence $\left(\epsilon_{i j} \gg \epsilon_{0}\right)$. These features make our haplotype inference method robust against the presence of ambiguous haplotype linkage from false variant sites and can implicitly exclude some of these variants based on haplotype linkage.

\section{Computational inference of the parental haplotypes of diploid NA12878 and RPE-1 genomes}

We first performed local haplotype inference using the linked-reads data. We restricted haplotype inference to bi-allelic singlenucleotide variant (SNV) sites as they represent the majority of genetic variation and have better detection accuracy than more complex alterations (insertion/deletion/structural variants). For each chromosome, we accumulated all molecular linkage between SNVs within $100 \mathrm{~kb}$ from each other and solved for the optimal haplotype by minimizing Eq. (10). The haplotype solution converged after 4-5 rounds of spin flipping and block switching. See SI Discussion and Data Table S1 for more technical details.

Phasing accuracy is assessed using the energy penalties calculated based on the final haplotype solution: The spin-flipping penalty (Eq. (11)) measures the probability of local ("short-switching") phasing errors; the block-switching penalty (Eq. (12)) measures the probability of long-range switching errors. Both penalty scores show bimodal distributions (Fig. S1). Sites with low spin-flipping penalty scores are enriched with false variants (Fig. S1A,D); by contrast, a large fraction of sites with low blockswitching penalty scores are found in regions of low variant density (Fig. S1C,F).

We can generate haplotype blocks with different levels of accuracy using different block-switching penalty cutoffs. This is illustrated in Fig. 4 using Chr.5 as an example for both samples. As expected, choosing lower block-switching cutoffs produces longer haplotype blocks with more intra-block switching errors than choosing higher cutoffs. The low-accuracy blocks (colored in red) usually contain only one or a few switching errors at sites with low block-switching penalty scores: these blocks are broken into two or more high-confidence blocks at a higher block-switching cutoff (red arrows). As the main goal of local haplotype inference here is to generate high-confidence blocks that can be concatenated by long-range Hi-C links, we have chosen conservative blockswitching cutoffs to produce short haplotype blocks with high accuracy. The cutoffs are determined based on the location of the minimum in the block-switching penalty distribution (Fig. S1B,E): $\Delta E 1000$ for the RPE-1 data and $\Delta E 5000$ for the NA12878 data. The resulting haplotype blocks are much shorter than reported previously (Marks et al. 2019), but the uniform accuracy (98\%) of each block ensures consistent accuracy across the entire chromosome once all the blocks are merged.

We note that haplotype blocks are often terminated at regions of low-variant density (less than 1 per $10 \mathrm{~kb}$ ). (Also see Figs. S1C,S1F,S2 and S3.) Two examples of large low-variant density regions are highlighted in the RPE-1 genome (black arrows in Fig. 4B). The first one in 5q13.2 is also seen in the NA12878 genome: This region, known as the spinal muscular atrophy (SMA) region, contains large segmental duplications ( 200kb) with high sequence similarity (98\%) (Schmutz et al., 2004) that cannot be resolved by short sequencing reads. Even though the standard variant caller (HaplotypeCaller) emits many variants in this region with variant density above 1 per $10 \mathrm{~kb}$ (blue tracks), most of these variants are false and left out in the reference haplotype (purple tracks). They are largely excluded from the final haplotype solution (green tracks) due to inconsistent or missing haplotype linkage. By contrast, the second low-variant density region in the RPE-1 genome near 5p21.1 contains few variants and most likely reflects loss-of-heterozygosity. The exclusion of both regions in the final haplotype solution confirms the robustness of our haplotype inference algorithm against false variants with inconsistent haplotype linkage. 
bioRxiv preprint doi: https://doi.org/10.1101/629337; this version posted June 21,2020 . The copyright holder for this preprint (which was not certified by peer review) is the author/funder, who has granted bioRxiv a license to display the preprint in perpetuity. It is made available under aCC-BY-NC-ND 4.0 International license.

We merge haplotype blocks using Hi-C links in two steps. First, haplotype blocks within each chromosome arm are concatenated using Hi-C links between variants separated by $\leq 10 \mathrm{Mb}$. Second, $\mathrm{p}$ - and q-arm haplotypes are joined using all Hi-C links between the arms. The consistency of haplotype solution in each step can be verified by comparing the number of cis and trans Hi-C links (Fig. S4). To expedite convergence of the haplotype solution, we exclude short haplotype blocks with $\leq 5$ total Hi-C links to other blocks from the calculation. We refer to the concatenated haplotype blocks as the "scaffold haplotype."

After generating the scaffold haplotype, we then calculate the linkage between variant genotypes and the scaffold haplotype using

$$
\begin{aligned}
& \eta_{\mathrm{rA}} \text { \#(reference-haplotype A) \#(alternate-haplotype B); } \\
& \eta_{\mathrm{rB}} \text { \#(reference-haplotype B) \#(alternate-haplotype A), }
\end{aligned}
$$

Here the counts reflect the number of unique molecules linking each genotype (Reference or Alternate) to either parental haplotype (A or B). We used the linkage to scaffold haplotypes to assign the haplotype phase at each variant site in the final haplotype solution.

\section{Variant filtration using haplotype linkage}

For true heterozygous variants, we expect the variant genotypes to be phased to different parental haplotypes: $\eta_{\mathrm{rA}} \gg \eta_{\mathrm{rB}} \approx 0$ or $\eta_{\mathrm{rB}} \gg \eta_{\mathrm{rA}} \approx 0$. We implemented a "linkage filter" to exclude false variants in the final haplotype solution based on the following
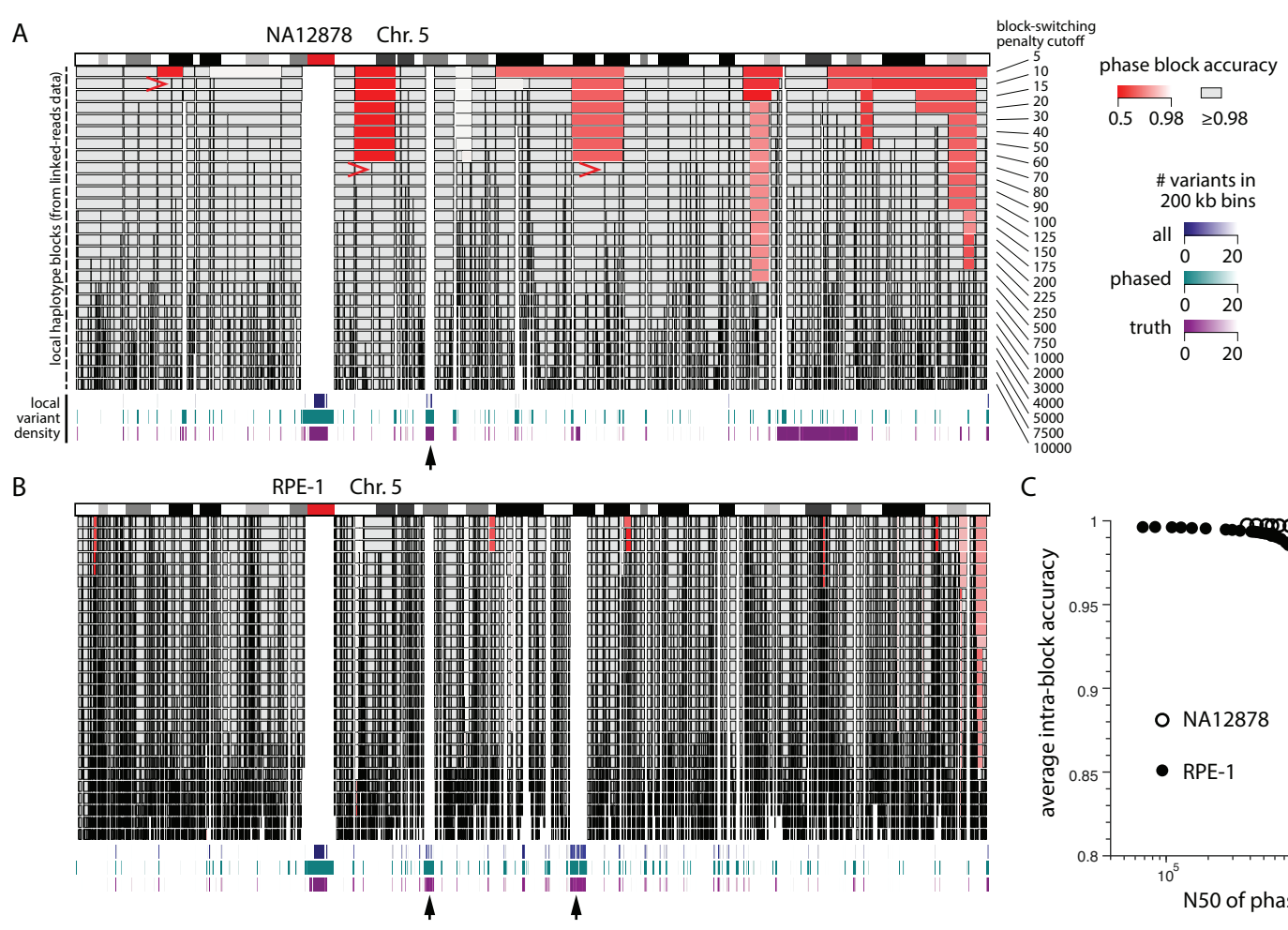

Figure 4: Haplotype blocks on Chr.5 inferred from the NA12878 and the RPE-1 linked-reads data. In panel 4A (NA12878) and B (RPE-1), each row represents haplotype blocks determined using a different switching-penalty cutoff (from $\Delta E 5$ to $\Delta E 10,000$ ). Only blocks with 50 or more phased variants are shown. The accuracy of each block is estimated by the percentage of genotypes that are consistent with the majority haplotype of each block determined using the reference haplotype. Blocks with $\geq 98 \%$ accuracy are colored in grey; those with $<98 \%$ accuracy are colored in red with brightness scaled by the accuracy. Three examples of lowaccuracy blocks that are broken into two separate high-accuracy blocks at a higher block-switching cutoff are highlighted by red arrows in the NA12878 genome. Shown below the haplotype blocks are three tracks of regional variant density corresponding to the number of total detected variants (blue), phased variants in the final haplotype solution (green), and phased variants in the reference data (purple) in $200 \mathrm{~kb}$ bins. Bins with more than 20 variants (variant density more than 1 per $10 \mathrm{~kb}$ ) are omitted. Black arrows highlight examples of large variant poor regions. Panel $\mathrm{C}$ shows the average intra-block accuracy (weighted by the number of variants in each block) and the N50 lengths of all haplotype blocks in each genome generated using different switching-penalty cutoffs. The NA12878 dataset produces longer haplotype blocks due to having longer input molecules (see Table 1 . 
criteria of haplotype linkage:

$$
\min \left(\eta_{\mathrm{rA}}, \eta_{\mathrm{rB}}\right) \leq \max \left[2,0.1 \times\left(\eta_{\mathrm{rA}} \eta_{\mathrm{rB}}\right)\right]
$$

with at least one link to both genotypes ( $\mathrm{R}$ and $\mathrm{A}$ ) and both scaffold haplotypes (A and $\mathrm{B}$ ).

Table 2: Comparison between the final haplotype solution and the reference haplotype of NA12878

\begin{tabular}{crrrrrrr} 
Total variant sites & $\begin{array}{r}\text { Phased from } \\
\text { bulk data }\end{array}$ & $\begin{array}{r}\text { Phased from } \\
\text { parental data }\end{array}$ & $\begin{array}{r}\text { Comparable } \\
\text { sites }\end{array}$ & Agreed & Accuracy & $\begin{array}{r}\text { Fraction of } \\
\text { completion }\end{array}$ \\
\hline $2,652,381$ & $2,319,02 \sqrt{a}$ & $1,861,941$ & $1,824,401$ & $1,818,042$ & 0.997 & 0.980 \\
& $2,151,64 \sqrt{b}$ & $1,815,212$ & $1,815,197$ & $1,809,886$ & 0.997 & 0.975 \\
\hline
\end{tabular}

\footnotetext{
${ }^{a}$ All phased variants without any filtering $\leq 0.1$

${ }^{b}$ Filtered by haplotype linkage: $\geq 1$ link connecting ref, alt, HapA, and HapB, and minor linkage $\leq 2$ or minor linkage/total linkage
}

Table 3: Comparison between the final haplotype solution and the reference haplotype of RPE-1

\begin{tabular}{|c|c|c|c|c|c|c|}
\hline Filter & ariant sites & $\begin{array}{r}\text { Phased from bulk } \\
\text { data }\end{array}$ & $\begin{array}{l}\text { Phased from } \\
\text { monosomies }\end{array}$ & Agreed & Discordant & $\begin{array}{l}\text { Fraction of } \\
\text { discordance }\end{array}$ \\
\hline None & $2,475,311$ & $2,242,237$ & $2,320,153$ & $2,101,195$ & 40,006 & 0.019 \\
\hline Allele fractior ${ }^{a}$ & $2,172,689$ & $2,087,188$ & $2,109,589$ & $2,018,906$ & 12,903 & 0.006 \\
\hline Linkage & $2,156,423$ & $2,156,346$ & $2,071,674$ & $2,054,006$ & 17,616 & 0.009 \\
\hline Combines ${ }^{b}$ & $2,054,859$ & $2,054,832$ & $2,001,674$ & $1,993,552$ & 8,098 & 0.004 \\
\hline
\end{tabular}

${ }^{a}$ From single-cell data: minor allele fraction $\geq 0.3$ in disomic regions and $\in[0.18,0.48]$ in the trisomic region in Chr.10q.

${ }^{b}$ With both the allele fraction (a) and the linkage (b) filter

\section{Benchmark of the haplotype solution}

We evaluated the accuracy and completeness of the computationally inferred haplotypes using independent reference haplotype data (Table 11). For the NA12878 genome, the reference haplotypes were determined from the parental genomes and released by the Genome-In-A-Bottle consortium. For the RPE-1 genome, we determined the reference haplotypes from the sequencing data of monosomic RPE-1 cells. The NA12878 haplotype data have high specificity but leave out several large regions including the parms of Chrs.16 and 18. These regions were excluded from benchmarking. For the reference RPE-1 haplotype data, we additionally implemented a variant filter based on the average variant allele fraction in all the single-cell data.

We first benchmarked the scaffold haplotype solution constructed from large haplotype blocks (Table S2 for the NA12878 dataset and Table S3 for the RPE-1 dataset). For the NA12878 sample, the scaffold haplotype solution contained $94 \%$ of all phased variants in the reference haplotype data with 99.6\% accuracy. (Chromosome 19 has the lowest accuracy of $98.5 \%$.) For the RPE-1 sample, the scaffold haplotype solution contained $89 \%$ of all phased variants in the reference haplotype data and showed $98.3 \%$ agreement. (Chromosome 9 has the lowest percentage of agreement of 96.1\%.) There is no long-range switching error in any chromosome in either sample.

We then benchmarked the final haplotype solution determined using the linkage between variant genotypes and the scaffold haplotypes. For the NA12878 sample, the final haplotype solution showed $99.7 \%$ accuracy and $98.0 \%$ completeness when compared to the reference haplotype (Table 2). The linkage filter removed 167,385 variants but did not affect the benchmarks as most of the false variants were already excluded from the reference haplotype.

For the RPE-1 sample, the final haplotype solution showed $98 \%$ agreement with the reference data before variant filtration (Table 3. We expected a large percentage of the discrepancy to be due to false variants. After excluding false variants based on either the variant allele fraction in the single cell data (100 samples) or the haplotype linkage in the linked-reads data, we saw $99 \%$ agreement between the haplotype solution and the reference haplotype. Although the linkage filter and the allele fraction filter are completely independent, they have good consistency: 2,054,859 variants pass both filters and represent $95 \%$ of variants passing each individual filter. Among variants passing both filters, the percentage of agreement between the haplotype solution and the reference haplotype is $99.6 \%$ and comparable to the NA12878 haplotype solution. 
bioRxiv preprint doi: https://doi.org/10.1101/629337; this version posted June 21,2020 . The copyright holder for this preprint (which was not certified by peer review) is the author/funder, who has granted bioRxiv a license to display the preprint in perpetuity. It is made available under aCC-BY-NC-ND 4.0 International license.

\section{Resolving chromosome-specific alterations using haplotype copy number}

To demonstrate this application, we use the parental RPE-1 haplotype information to resolve large structural alterations of chromosomes in RPE-1 cells generated by telomere fusions (Maciejowski et al. 2015). In this study, the authors performed bulk whole-genome sequencing on the progeny populations of single RPE-1 cells that underwent telomere crisis. We downloaded and reprocessed the sequencing data using our standard workflow and calculated haplotype coverage in 250kb bins using the RPE-1
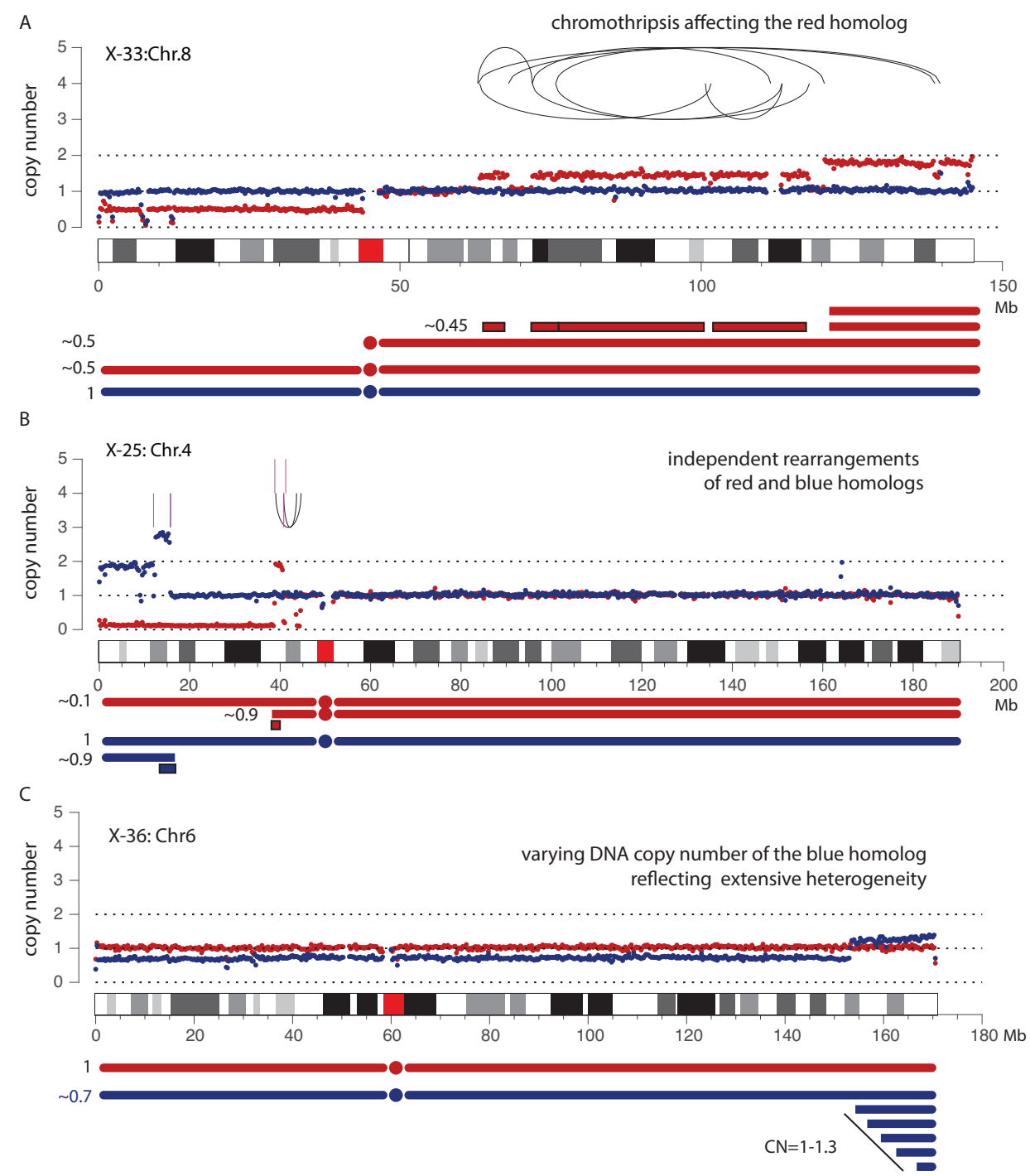

Figure 5: Haplotype-resolved DNA copy number and rearrangement analysis of post-crisis RPE-1 cells from Ref. Maciejowski et al. (2015). Schematic diagrams of alterations to each homolog and their clonal fractions are shown below the copy-number plots. Non-telomeric chromosomal fragments involved in complex rearrangements are outlined. A. Haplotype-specific DNA copy number (blue and red dots for each parental haplotype) and rearrangement (black arcs) of Chr.8 in the X-33 sample. Both the rearrangements and the copy-number alterations are restricted to the red homolog; the non-integer copy-number states indicate that the altered Chr.8 is present in a subclonal population ( 45\%). B. Haplotype-specific DNA copy number and rearrangement of Chr.4 (black arcs: intra-chromosomal; magenta vertical lines: inter-chromosomal to Chr.13) in the X-25 sample. Rearrangements and copy-number alterations affect both homologs. Both the gain of the blue homolog and the loss of the red homolog appear to be subclonal ( 90\%). C. Haplotype-specific copy number of Chr.6 in the X-36 sample. The q-terminus of the blue homolog shows non-constant copy number (1-1.3) that contrasts with the stable copy number of the red homolog or the rest of the blue homolog $(\sim 0.7)$. We interpret this pattern as reflecting extensive genetic heterogeneity in the population. 
haplotype data as

$$
C_{A, B}^{(i)} D^{(i)} \cdot \overline{R_{A, B}^{(i)}}
$$

where $D^{(i)}$ is the normalized average sequence coverage in the $i$ th bin $\overline{D^{(i)}}$ 1) and $\overline{R_{A, B}^{(i)}}$ is the mean haplotype fraction (A or B) across all variants within the $i$ th bin. We then calculated haplotype DNA copy number by normalizing $C_{A, B}$ by its median value across the whole genome (from both haplotypes). The median value corresponds to the expected (normalized) coverage of a single chromosome (haplotype) assuming the genome is mostly diploid.

Figure 6 shows three examples of complex chromosomal alterations, each taken from a different sample that underwent telomere crisis. Haplotype-specific copy number is shown using red and blue dots; chromosomal rearrangements accompanying the copy-number alterations are shown as black arcs (intra-chromosomal events) and magenta vertical lines (breakpoints of interchromosomal translocations). The first example (Fig. 64) shows a chromothripsis event affecting the 8q arm of the red homolog. Based on the non-integer copy-number states of the red haplotype and the near diploid karyotype of this sample (Maciejowski et al. 2015), we infer that the altered Chr.8q is present in a subclonal population ( 45\%). The second example (Fig. 6B) shows alterations to both Chr.4 homologs on the p-arm: Both the gain of the blue haplotype and the loss of the red haplotype are subclonal ( $\sim 90 \%)$; the broken ends on both homologs are linked to Chr.13 (magenta lines), suggesting a complex event involving three separate chromosomes. The last example (Fig. 6C) shows an intriguing pattern of copy-number variation. The varying copy number of the blue haplotype at the q-terminus contrasts with the constant copy number of the red haplotype or the rest of the blue haplotype, excluding the possibility that the variation is due to technical artifacts. We interpret this varying copy number pattern as the outcome of varying terminal segments present in different cells in the population (Umbreit et al., 2020).

The haplotype copy-number analysis demonstrates that the progeny populations of single cells passing through telomere crisis can be highly heterogeneous. The feature of non-constant haplotype copy number is of particular interest and may be used as a signature to infer ongoing genome instability in a cell population directly from bulk DNA sequencing.

\section{Walking derivate chromosomes using haplotype-specific Hi-C contacts}

Hi-C sequencing has previously been used for detecting long-range chromosomal rearrangements (Rao et al. 2014 , Dixon et al. 2018; Zhou et al. 2019). The formation of new junctions between distal loci (separated by 1 Mb genomic distance or located on different chromosomes) creates new cis contacts with a significantly higher density than trans contacts in a normal genome. As each rearrangement breakpoint is generated on one parental chromosome, the newly formed cis contacts at the rearrangement junction should be phased to one haplotype on either side of the junction. For inter-chromosomal rearrangements, cis contacts between the parter chromosomes should be observed in one out of four possible haplotype combinations (AA, AB, BA, or BB); for intra-chromosomal rearrangements, newly formed cis contacts should be observed in one out of three possible combinations (AA, $\mathrm{AB}$, or $\mathrm{BB})$. Combining haplotype-specific connectivity from Hi-C contacts with haplotype DNA copy number from linked-reads data enables us to infer the syntenic structure of derivative chromosomes and generate phased karyotypes (Fig. 6.

We first illustrate this approach using a simple example in the RPE-1 genome (Fig. 6A). RPE-1 cells contain an extra copy of a segment from Chr.10q (62 Mb-qter). The DNA sequence near the breakpoint on Chr.10q shows repeats whose origin cannot be determined; cytogenetic analysis indicates that this segment is fused to the q-terminus of Chr.X. In the phased Hi-C contact map, this translocation is easily recognized from the enrichment of contacts near the q-terminus of Chr.X and the breakpoint on Chr.10q ( 62Mb) that is restricted to one haplotype combination (arbitrarily denoted as A for both chromosomes). Importantly, the enrichment of Hi-C contacts extends throughout Chr.X to the p-terminus, indicating that the 10q segment joins a complete $\mathrm{X}$ chromosome and confirming the result from cytogenetic analysis.

Using a similar strategy, we are able to generate a "digital karyotype" of the K-562 genome using published sequencing data (Table S1). The K-562 genome is highly aneuploid (Zhou et al., 2019) and contains multiple marker chromosomes (Gribble et al. 2000: Naumann et al. 2001) and large regions of loss-of-heterozygosity (LOH). We determined the parental haplotypes in heterozygous regions and then calculated haplotype-specific DNA copy number using phased links in the linked-reads data and generated haplotype-specific Hi-C maps using Hi-C contacts that are phased on at least one end. The digital karyotype was determined by a joint analysis of haplotype-specific DNA copy number, rearrangements, and Hi-C contacts (SI Additional Data). The digital karyotype is schematically shown in Fig. 6C and shows excellent agreement with the cytogenetic karyotype reported in Ref. (Gribble et al. 2000) (Fig. 6B) and (Naumann et al. 2001). The digital karyotype shows excellent agreement with the cytogenetic karyotype but provides haplotype resolution of the syntenic blocks and base-pair resolution of the translocation junctions in 9 marker chromosomes reported in Ref. (Gribble et al. 2000) (outlined in Fig. 63 and schematically shown in Fig. 6F) and 3 additional marker chromosomes in (Naumann et al. 2001). We are also able to partially resolve the structure of the complex amplicon containing the BCR-ABL fusion in $\mathrm{t}(22 \mathrm{~A} ; 9-13-22 \mathrm{hsr})$ combining sequencing and cytogenetic data. Details of the reconstructed marker chromosomes are provided in SI additional Data. 


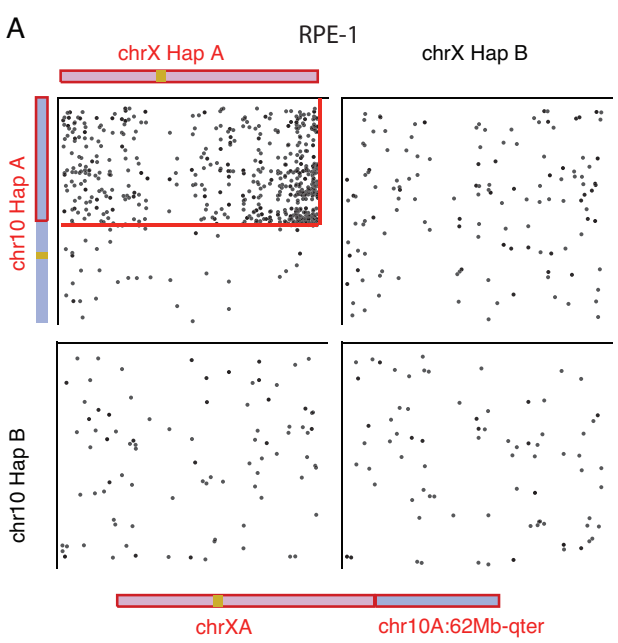

B

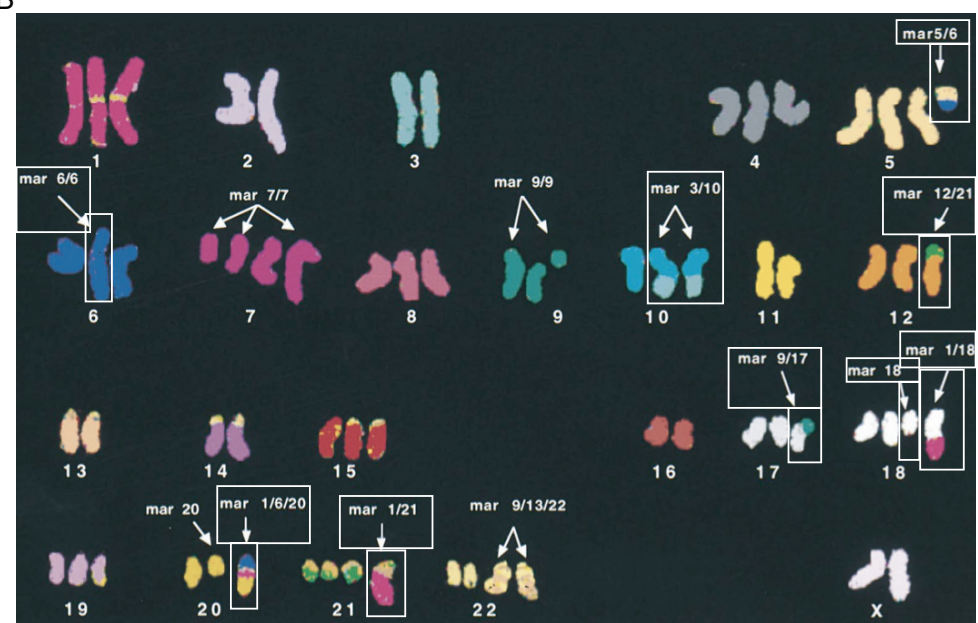

C
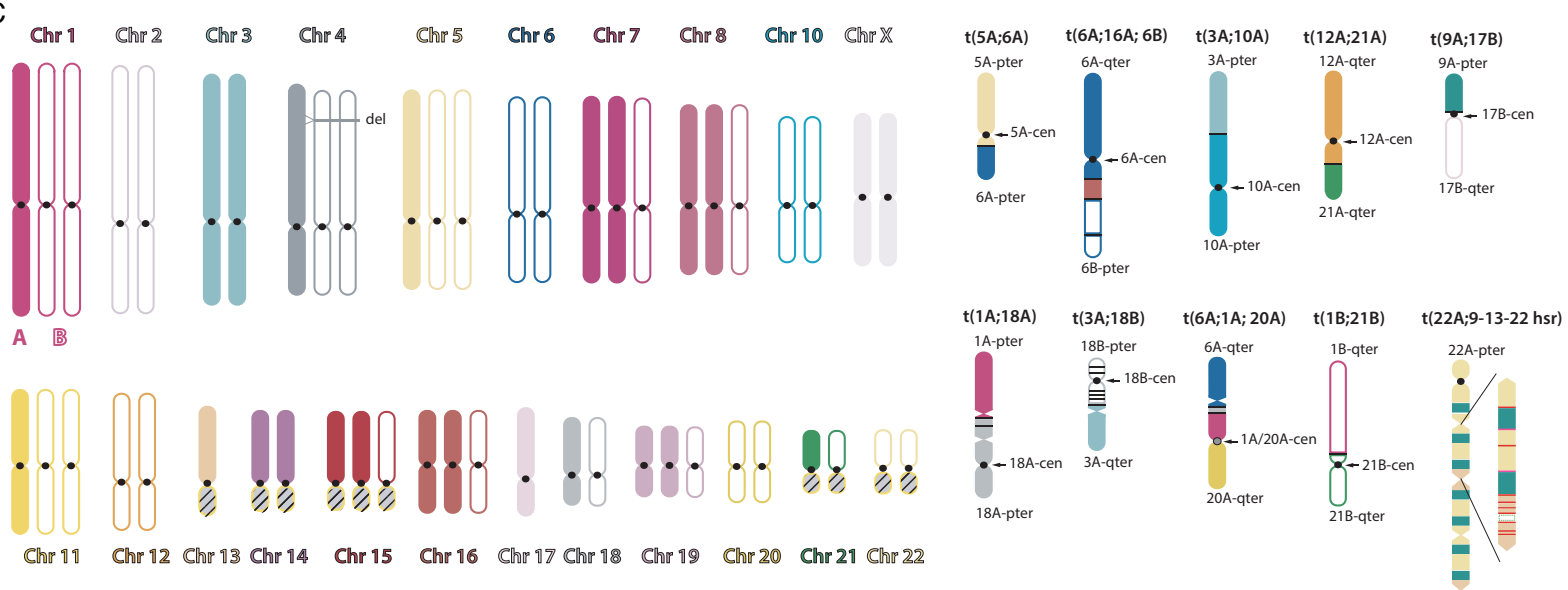

Figure 6: Haplotype-resolved structure of derivative chromosomes and aneuploid karyotypes. A. Walking the translocated X chromosome in the RPE-1 genome using phased Hi-C links (dots) between different homologs of Chr.10 and Chr.X. A significant enrichment of Hi-C links is seen in only one haplotype combination reflecting the translocation between Chr.10 and Chr.X. The enrichment of Hi-C links throughout the entire $\mathrm{X}$ chromosome suggests the composition of the derivative chromosome as shown at the bottom. B. The cytogenetic K-562 karyotype reported in Ref. (Gribble et al., 2000) with outlined marker chromosomes resolved by sequencing data and shown in C. C. The digital K-562 karyotype determined from linked-reads and Hi-C sequencing data (Table S1), with haplotype assignment to both normal (left) and marker (right) chromosomes. The digital karyotype mostly agrees with the cytogenetic karyotype and the differences may be attributed to additional alterations during cell culture. Among all the marker chromosomes listed in Panel B, we are able to determine the syntenic structure of the following: mar5/6, mar6/6, mar3/10, mar12/21, mar9/17, mar1/18, mar1/6/20, and mar1/21, and resolve most translocation junctions at the base pair level. Multiple junctions contained local fold-back rearrangements (opposite arrows in $\mathrm{t}(1 \mathrm{~A} ; 18 \mathrm{~A}), \mathrm{t}(3 \mathrm{~A} ; 18 \mathrm{~B}), \mathrm{t}(6 \mathrm{~A} ; 1 \mathrm{~A} ; 20 \mathrm{~A}))$ that are consistent with local DNA copy number gains; these events cannot be resolved by cytogenetic analyses. The mar18 described in Ref. (Gribble et al. 2000) is probably related/similar to $\mathrm{t}(3 \mathrm{~A} ; 18 \mathrm{~B})$. The $B C R-A B L$ amplification is contained in a homogeneously staining region (hsr) of a marker chromosome $\mathrm{t}(22 \mathrm{~A} ; 9-13-22 \mathrm{hsr})$. We infer the structure of the amplicon from DNA copy number and rearrangements but cannot validate the inferred structure due to technical limitations. We are further able to partially determine the structures of the altered Chr.7 and Chr.9 and resolve three additional marker chromosomes described in Ref. (Naumann et al. 2001) but not in Ref. (Gribble et al. 2000): t(2A;22A), t(3A;10A;17A), t(9A;13A). These results are summarized in Additional Dataset 2.

\section{Discussion}

Here we describe a computational method that can accurately determine complete chromosomal haplotypes from a combination of linked-reads sequencing (30-60× mean depth) and Hi-C sequencing ( $\geq 50$ million long-range contacts). The computationally 
inferred haplotypes show high accuracy (99\%) and completeness (98\%) when compared to independent reference haplotypes.

Our method offers several advantages over previous methods. First, both linked-reads and Hi-C sequencing data can be generated on standard sequencing platforms and the construction of sequencing libraries does not involve special experimental techniques required for single-chromosome isolation (Ma et al., 2010, Fan et al. 2010, Yang et al. 2010), single-cell sequencing (Zhang et al. 2015), or similar techniques such as "Strand-Seq" (Falconer et al. 2012; Porubsky et al. 2017). Second, the computational algorithm implicitly excludes inconsistent linkage evidence from false variants based on the specificity of haplotype linkage. This contrasts with previous methods (Edge et al. 2017) that require high-quality variants as input. Our method further enables a variantfiltering strategy based on haplotype linkage that can be used to exclude false variants due to alignment errors and validate complex variants such as small insertions and deletions, or large structural variants.

Our formalism of haplotype inference as a minimization problem also has several unique features. The symmetric representation of binary genotypes and haplotypes simplifies the inference of complementary haplotypes into one minimization problem based on linkage evidence from both parental chromosomes. The haplotype inference algorithm is not affected by allelic imbalance, including loss-of-heterozygosity, and is directly applicable to aneuploid tumor genomes. We demonstrate that a simple iteration strategy can efficiently solve the parental haplotypes of diploid genomes but it is straightforward to incorporate more sophisticated minimization algorithms (e.g., Monte-Carlo methods) when necessary. One useful extension of our method is to perform joint haplotype inference using population genotypes and Hi-C data. Population-based statistical phasing (Loh et al. 2016a) can produce long haplotype blocks $(1 \mathrm{Mb})$ that contain random but rare switching errors. It should be possible to correct these errors using Hi-C data and extend the statistical haplotype phase to the entire chromosome.

Knowledge of chromosomal haplotypes can be used to directly relate variations in the DNA sequence, histone marks, chromatin structure, and gene expression on each chromosome. This is especially useful for the analysis of cancer genomes where homologous chromosomes often acquire independent alterations in the DNA sequence that can cause differential changes in chromatin folding and gene expression (Spielmann et al. 2018). We demonstrate the capability to resolve the composition of derivative chromosomes and aneuploid genomes by constructing a digital karyotype of the K-562 genome from linked-reads and Hi-C sequencing data. We expect this strategy to be generally applicable to complex cancer genomes. The capability to determine the large-scale structure of derivative chromosomes shall enable us to further investigate the connection between 2D chromosomal structural alterations and $3 \mathrm{D}$ chromatin reorganization.

\section{References}

Adey, A., Burton, J. N., Kitzman, J. O., Hiatt, J. B., Lewis, A. P., Martin, B. K., Qiu, R., Lee, C., and Shendure, J. (2013a). The haplotype-resolved genome and epigenome of the aneuploid hela cancer cell line. Nature, 500(7461):207-211.

Adey, A., Kitzman, J. O., Burton, J. N., Daza, R., Kumar, A., Christiansen, L., Ronaghi, M., Amini, S., L Gunderson, K., Steemers, F. J., and Shendure, J. (2014). In vitro, long-range sequence information for de novo genome assembly via transposase contiguity. Genome Research, 24(12):2041-2049.

Adey, A., Patwardhan, R. P., Qiu, R., Kitzman, J. O., Burton, J. N., and Shendure, J. (2013b). Chromosome-scale scaffolding of de novo genome assemblies based on chromatin interactions. Nature Biotechnology, 31(12):1119-1125.

Browning, S. R. and Browning, B. L. (2007). Rapid and accurate haplotype phasing and missing-data inference for whole-genome association studies by use of localized haplotype clustering. The American Journal of Human Genetics, 81(5):1084-1097.

Browning, S. R. and Browning, B. L. (2011). Haplotype phasing: existing methods and new developments. Nature Reviews Genetics, 12(10):703-714.

Chu, W. K., Edge, P., Lee, H. S., Bansal, V., Bafna, V., Huang, X., and Zhang, K. (2017). Ultraaccurate genome sequencing and haplotyping of single human cells. Proceedings of the National Academy of Sciences, 114(47):12512-12517.

Daly, M. J., Rioux, J. D., Schaffner, S. F., Hudson, T. J., and Lander, E. S. (2001). High-resolution haplotype structure in the human genome. Nature Genetics, 29(2):229-232.

Darrow, E. M., Huntley, M. H., Dudchenko, O., Stamenova, E. K., Durand, N. C., Sun, Z., Huang, S.-C., Sanborn, A. L., Machol, I., Shamim, M., Seberg, A. P., Lander, E. S., Chadwick, B. P., and Aiden, E. L. (2016). Deletion of DXZ4on the human inactive X chromosome alters higher-order genome architecture. Proceedings of the National Academy of Sciences, 113(31):E4504-E4512.

Deng, Q., Ramskold, D., Reinius, B., and Sandberg, R. (2014). Single-cell rna-seq reveals dynamic, random monoallelic gene expression in mammalian cells. Science, 343(6167):193-196. 
Dixon, J. R., Xu, J., Dileep, V., Zhan, Y., Song, F., Le, V. T., Yardımc1, G. G., Chakraborty, A., Bann, D. V., Wang, Y., Clark, R., Zhang, L., Yang, H., Liu, T., Iyyanki, S., An, L., Pool, C., Sasaki, T., Rivera-Mulia, J. C., Ozadam, H., Lajoie, B. R., Kaul, R., Buckley, M., Lee, K., Diegel, M., Pezic, D., Ernst, C., Hadjur, S., Odom, D. T., Stamatoyannopoulos, J. A., Broach, J. R., Hardison, R. C., Ay, F., Noble, W. S., Dekker, J., Gilbert, D. M., and Yue, F. (2018). Integrative detection and analysis of structural variation in cancer genomes. Nature Genetics, 50(10):1388-1398.

Edge, P., Bafna, V., and Bansal, V. (2017). HapCUT2: robust and accurate haplotype assembly for diverse sequencing technologies. Genome Research, 27(5):801-812.

Falconer, E., Hills, M., Naumann, U., Poon, S. S. S., Chavez, E. A., Sanders, A. D., Zhao, Y., Hirst, M., and Lansdorp, P. M. (2012). DNA template strand sequencing of single-cells maps genomic rearrangements at high resolution. Nature Methods, 9(11):1107-1112.

Fan, H. C., Wang, J., Potanina, A., and Quake, S. R. (2010). Whole-genome molecular haplotyping of single cells. Nature Biotechnology, 29(1):51-57.

Gribble, S. M., Roberts, I., Grace, C., Andrews, K. M., Green, A. R., and Nacheva, E. P. (2000). Cytogenetics of the chronic myeloid leukemia-derived cell line k562: karyotype clarification by multicolor fluorescence in situ hybridization, comparative genomic hybridization, and locus-specific fluorescence in situ hybridization. Cancer Genet Cytogenet, 118(1):1-8.

Hansen, C. H. and van Oudenaarden, A. (2013). Allele-specific detection of single mrna molecules in situ. Nature Methods, 10(9):869-871.

Kaper, F., Swamy, S., Klotzle, B., Munchel, S., Cottrell, J., Bibikova, M., Chuang, H.-Y., Kruglyak, S., Ronaghi, M., Eberle, M. A., and Fan, J.-B. (2013). Whole-genome haplotyping by dilution, amplification, and sequencing. Proc. Natl. Acad. Sci. U.S.A., 110(14):5552-5557.

Kitzman, J. O., MacKenzie, A. P., Adey, A., Hiatt, J. B., Patwardhan, R. P., Sudmant, P. H., Ng, S. B., Alkan, C., Qiu, R., Eichler, E. E., and Shendure, J. (2010). Haplotype-resolved genome sequencing of a Gujarati Indian individual. Nature Biotechnology, 29(1):59-63.

Kong, A., Masson, G., Frigge, M. L., Gylfason, A., Zusmanovich, P., Thorleifsson, G., Olason, P. I., Ingason, A., Steinberg, S., Rafnar, T., Sulem, P., Mouy, M., Jonsson, F., Thorsteinsdottir, U., Gudbjartsson, D. F., Stefansson, H., and Stefansson, K. (2008). Detection of sharing by descent, long-range phasing and haplotype imputation. Nature Genetics, 40(9):1068-1075.

Levesque, M. J. and Raj, A. (2013). Single-chromosome transcriptional profiling reveals chromosomal gene expression regulation. Nature Methods, 10(3):246-248.

Lieberman-Aiden, E., van Berkum, N. L., Williams, L., Imakaev, M., Ragoczy, T., Telling, A., Amit, I., Lajoie, B. R., Sabo, P. J., Dorschner, M. O., and et al. (2009). Comprehensive mapping of long-range interactions reveals folding principles of the human genome. Science, 326(5950):289-293.

Loh, P.-R., Danecek, P., Palamara, P. F., Fuchsberger, C., A Reshef, Y., K Finucane, H., Schoenherr, S., Forer, L., McCarthy, S., Abecasis, G. R., Durbin, R., and L Price, A. (2016a). Reference-based phasing using the Haplotype Reference Consortium panel. Nature Genetics, 48(11):1443-1448.

Loh, P.-R., Genovese, G., Handsaker, R. E., Finucane, H. K., Reshef, Y. A., Palamara, P. F., Birmann, B. M., Talkowski, M. E., Bakhoum, S. F., McCarroll, S. A., and Price, A. L. (2018). Insights into clonal haematopoiesis from 8,342 mosaic chromosomal alterations. Nature Publishing Group, pages 1-22.

Loh, P.-R., Palamara, P. F., and Price, A. L. (2016b). Fast and accurate long-range phasing in a UK Biobank cohort. Nature Genetics, 48(7):811-816.

Ma, L., Xiao, Y., Huang, H., Wang, Q., Rao, W., Feng, Y., Zhang, K., and Song, Q. (2010). Direct determination of molecular haplotypes by chromosome microdissection. Nature Publishing Group, 7(4):299-301.

Maciejowski, J., Li, Y., Bosco, N., Campbell, P. J., and de Lange, T. (2015). Chromothripsis and kataegis induced by telomere crisis. Cell, 163(7):1641 - 1654 . 
Marks, P., Garcia, S., Barrio, A. M., Belhocine, K., Bernate, J., Bharadwaj, R., Bjornson, K., Catalanotti, C., Delaney, J., Fehr, A., Fiddes, I. T., Galvin, B., Heaton, H., Herschleb, J., Hindson, C., Holt, E., Jabara, C. B., Jett, S., Keivanfar, N., KyriazopoulouPanagiotopoulou, S., Lek, M., Lin, B., Lowe, A., Mahamdallie, S., Maheshwari, S., Makarewicz, T., Marshall, J., Meschi, F., O’Keefe, C. J., Ordonez, H., Patel, P., Price, A., Royall, A., Ruark, E., Seal, S., Schnall-Levin, M., Shah, P., Stafford, D., Williams, S., Wu, I., Xu, A. W., Rahman, N., MacArthur, D., and Church, D. M. (2019). Resolving the full spectrum of human genome variation using Linked-Reads. Genome Research, 29(4):635-645.

Naumann, S., Reutzel, D., Speicher, M., and Decker, H. J. (2001). Complete karyotype characterization of the k562 cell line by combined application of g-banding, multiplex-fluorescence in situ hybridization, fluorescence in situ hybridization, and comparative genomic hybridization. Leuk Res, 25(4):313-22.

Nik-Zainal, S., Van Loo, P., Wedge, D. C., Alexandrov, L. B., Greenman, C. D., Lau, K. W., Raine, K., Jones, D., Marshall, J., Ramakrishna, M., and et al. (2012). The life history of 21 breast cancers. Cell, 149(5):994-1007.

Pendleton, M., Sebra, R., Pang, A. W. C., Ummat, A., Franzen, O., Rausch, T., Stütz, A. M., Stedman, W., Anantharaman, T., Hastie, A., Dai, H., Fritz, M. H.-Y., Cao, H., Cohain, A., Deikus, G., Durrett, R. E., Blanchard, S. C., Altman, R., Chin, C.-S., Guo, Y., Paxinos, E. E., Korbel, J. O., Darnell, R. B., McCombie, W. R., Kwok, P.-Y., Mason, C. E., Schadt, E. E., and Bashir, A. (2015). Assembly and diploid architecture of an individual human genome via single-molecule technologies. Nature Methods, 12(8):780-786.

Peters, B. A., Kermani, B. G., Sparks, A. B., Alferov, O., Hong, P., Alexeev, A., Jiang, Y., Dahl, F., Tang, Y. T., Haas, J., Robasky, K., Zaranek, A. W., Lee, J.-H., Ball, M. P., Peterson, J. E., Perazich, H., Yeung, G., Liu, J., Chen, L., Kennemer, M. I., Pothuraju, K., Konvicka, K., Tsoupko-Sitnikov, M., Pant, K. P., Ebert, J. C., Nilsen, G. B., Baccash, J., Halpern, A. L., Church, G. M., and Drmanac, R. (2014). Accurate whole-genome sequencing and haplotyping from 10 to 20 human cells. Nature, 487(7406):190195.

Porubsky, D., Garg, S., Sanders, A. D., Korbel, J. O., Guryev, V., Lansdorp, P. M., and Marschall, T. (2017). Dense and accurate whole-chromosome haplotyping of individual genomes. Nature Communications, 8(1).

Rao, S. S. P., Huntley, M. H., Durand, N. C., Stamenova, E. K., Bochkov, I. D., Robinson, J. T., Sanborn, A. L., Machol, I., Omer, A. D., Lander, E. S., and Aiden, E. L. (2014). A 3d map of the human genome at kilobase resolution reveals principles of chromatin looping. Cell, 159(7):1665-80.

Reich, D. E., Cargill, M., Bolk, S., Ireland, J., Sabeti, P. C., Richter, D. J., Lavery, T., Kouyoumjian, R., Farhadian, S. F., Ward, R., and Lander, E. S. (2001). Linkage disequilibrium in the human genome. Nature, 411(6834):199-204.

Sanborn, A. L., Rao, S. S. P., Huang, S.-C., Durand, N. C., Huntley, M. H., Jewett, A. I., Bochkov, I. D., Chinnappan, D., Cutkosky, A., Li, J., and et al. (2015). Chromatin extrusion explains key features of loop and domain formation in wild-type and engineered genomes. Proceedings of the National Academy of Sciences, 112(47):E6456-E6465.

Scheet, P. and Stephens, M. (2006). A fast and flexible statistical model for large-scale population genotype data: Applications to inferring missing genotypes and haplotypic phase. The American Journal of Human Genetics, 78(4):629-644.

Schmutz, J., Martin, J., Terry, A., Couronne, O., Grimwood, J., Lowry, S., Gordon, L. A., Scott, D., Xie, G., Huang, W., Hellsten, U., Tran-Gyamfi, M., She, X., Prabhakar, S., Aerts, A., Altherr, M., Bajorek, E., Black, S., Branscomb, E., Caoile, C., Challacombe, J. F., Chan, Y. M., Denys, M., Detter, J. C., Escobar, J., Flowers, D., Fotopulos, D., Glavina, T., Gomez, M., Gonzales, E., Goodstein, D., Grigoriev, I., Groza, M., Hammon, N., Hawkins, T., Haydu, L., Israni, S., Jett, J., Kadner, K., Kimball, H., Kobayashi, A., Lopez, F., Lou, Y., Martinez, D., Medina, C., Morgan, J., Nandkeshwar, R., Noonan, J. P., Pitluck, S., Pollard, M., Predki, P., Priest, J., Ramirez, L., Retterer, J., Rodriguez, A., Rogers, S., Salamov, A., Salazar, A., Thayer, N., Tice, H., Tsai, M., Ustaszewska, A., Vo, N., Wheeler, J., Wu, K., Yang, J., Dickson, M., Cheng, J.-F., Eichler, E. E., Olsen, A., Pennacchio, L. A., Rokhsar, D. S., Richardson, P., Lucas, S. M., Myers, R. M., and Rubin, E. M. (2004). The dna sequence and comparative analysis of human chromosome 5. Nature, 431(7006):268-74.

Selvaraj, S., Dixon, J. R., Bansal, V., and Ren, B. (2013). Whole-genome haplotype reconstruction using proximity-ligation and shotgun sequencing. Nature Biotechnology, 31(12):1111-1118.

Seo, J.-S., Rhie, A., Kim, J., Lee, S., Sohn, M.-H., Kim, C.-U., Hastie, A., Cao, H., Yun, J.-Y., Kim, J., Kuk, J., Park, G. H., Kim, J., Ryu, H., Kim, J., Roh, M., Baek, J., Hunkapiller, M. W., Korlach, J., Shin, J.-Y., and Kim, C. (2016). De novo assembly and phasing of a Korean human genome. Nature Publishing Group, pages 1-18. 
Shendure, J. and Aiden, E. L. (2012). The expanding scope of DNA sequencing. Nature Publishing Group, 30(11):1084-1094.

Snyder, M. W., Adey, A., Kitzman, J. O., and Shendure, J. (2015). Haplotype-resolved genome sequencing: experimental methods and applications. Nature Reviews Genetics, 16(6):344-358.

Spielmann, M., Lupiáñez, D. G., and Mundlos, S. (2018). Structural variation in the 3d genome. Nature Reviews Genetics, pages $1-15$.

Suk, E. K., McEwen, G. K., Duitama, J., Nowick, K., Schulz, S., Palczewski, S., Schreiber, S., Holloway, D. T., McLaughlin, S., Peckham, H., Lee, C., Huebsch, T., and Hoehe, M. R. (2011). A comprehensively molecular haplotype-resolved genome of a European individual. Genome Research, 21(10):1672-1685.

The International HapMap Consortium (2005). A haplotype map of the human genome. Nature, 437(7063):1299-1320.

Umbreit, N. T., Zhang, C.-Z., Lynch, L. D., Blaine, L. J., Cheng, A. M., Tourdot, R., Sun, L., Almubarak, H. F., Judge, K., Mitchell, T. J., Spektor, A., and Pellman, D. (2020). Mechanisms generating cancer genome complexity from a single cell division error. Science, 368(6488).

Yang, H., Chen, X., and Wong, W. H. (2010). Completely phased genome sequencing through chromosome sorting. Proceedings of the National Academy of Sciences, 108(1):12-17.

Zhang, C.-Z. and Pellman, D. (2015). From mutational mechanisms in single cells to mutational patterns in cancer genomes. Cold Spring Harbor Symposia on Quantitative Biology, 80:117-137.

Zhang, C.-Z., Spektor, A., Cornils, H., Francis, J. M., Jackson, E. K., Liu, S., Meyerson, M., and Pellman, D. (2015). Chromothripsis from dna damage in micronuclei. Nature, 522(7555):179-184.

Zheng, G. X. Y., Lau, B. T., Schnall-Levin, M., Jarosz, M., Bell, J. M., Hindson, C. M., Kyriazopoulou-Panagiotopoulou, S., Masquelier, D. A., Merrill, L., Terry, J. M., Mudivarti, P. A., Wyatt, P. W., Bharadwaj, R., Makarewicz, A. J., Li, Y., Belgrader, P., Price, A. D., Lowe, A. J., Marks, P., Vurens, G. M., Hardenbol, P., Montesclaros, L., Luo, M., Greenfield, L., Wong, A., Birch, D. E., Short, S. W., Bjornson, K. P., Patel, P., Hopmans, E. S., Wood, C., Kaur, S., Lockwood, G. K., Stafford, D., Delaney, J. P., Wu, I., Ordonez, H. S., Grimes, S. M., Greer, S., Lee, J. Y., Belhocine, K., Giorda, K. M., Heaton, W. H., McDermott, G. P., Bent, Z. W., Meschi, F., Kondov, N. O., Wilson, R., Bernate, J. A., Gauby, S., Kindwall, A., Bermejo, C., Fehr, A. N., Chan, A., Saxonov, S., Ness, K. D., Hindson, B. J., and Ji, H. P. (2016). Haplotyping germline and cancer genomes with high-throughput linked-read sequencing. Nature Biotechnology, 34(3):303-311.

Zhou, B., Ho, S. S., Greer, S. U., Zhu, X., Bell, J. M., Arthur, J. G., Spies, N., Zhang, X., Byeon, S., Pattni, R., Ben-Efraim, N., Haney, M. S., Haraksingh, R. R., Song, G., Ji, H. P., Perrin, D., Wong, W. H., Abyzov, A., and Urban, A. E. (2019). Comprehensive, integrated, and phased whole-genome analysis of the primary ENCODE cell line K562. Genome Research, 29(3):472-484. 